\title{
MODULATION OF MOTOR CORTEX EXCITABILITY BY PAIRED PERIPHERAL AND TRANSCRANIAL MAGNETIC STIMULATION
}

Hatice Kumru, M.D., Ph.D. ${ }^{\mathrm{a}, \mathrm{b}, \mathrm{c},}$; Sergiu Albu, M.D., Ph.D ${ }^{\mathrm{d},}$, John Rothwell,PhD ${ }^{\mathrm{e}}$; Daniel Leon, M.D. ${ }^{\mathrm{a}, \mathrm{b}, \mathrm{c}}$; Cecilia Flores , M.D. ${ }^{\mathrm{a}, \mathrm{b}, \mathrm{c}}$, Eloy Opisso, M.D., Ph.D. ${ }^{\mathrm{a}, \mathrm{b}, \mathrm{c}}$, Josep Maria Tormos. M.D., Ph.D. ${ }^{\text {a,b,c }}$, Josep Valls-Sole, M.D., Ph.D. ${ }^{\text {f }}$

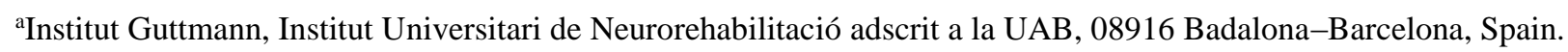

${ }^{\mathrm{b}}$ Universidad Autonoma de Barcelona, 08193 Bellaterra (Cerdanyola del Vallès), Spain

${ }^{c}$ Fundació Institut d'Investigació en Ciències de la Salut Germans Trias i Pujol, Badalona, Barcelona, Spain

${ }^{\mathrm{d}}$ Department of Psychology, Texas and M University, College Station, TX 77843, USA

London

${ }^{\mathrm{e}}$ Institute of Neurology, University College London, London WC1N 3BG, United Kingdom.

${ }^{\mathrm{f}} \mathrm{EMG}$ unit,Neurology, Hospital Clinic-Barcelona, Spain

Correspondence to: Hatice Kumru, MD, PhD.

Hospital de Neurorehabilitació Institut Guttmann

Camí Can Ruti s/n. Barcelona

08916 Barcelona, Spain.

Tel. + 349349777 00; Fax +34 934977715

Email: hkumru@guttmann.com 


\section{Highlights}

- We used for the first time combined repetitive peripheral magnetic stimulation and transcranial magnetic stimulation (magnetic-paired associated stimulation, magnetic-PAS).

- Magnetic-PAS modulates corticospinal excitability and cortical inhibition in the stimulated muscle.

- Application of magnetic-PAS may be a therapeutic option for motor rehabilitation. 


\section{ABSTRACT}

Objective: Repetitive application of peripheral electrical stimuli paired with transcranial magnetic stimulation (rTMS) of M1 cortex at low frequency, known as paired associative stimulation (PAS), is an effective method to induce motor cortex plasticity in humans. Here we investigated the effects of repetitive peripheral magnetic stimulation (rPMS) combined with low frequency rTMS ('magnetic-PAS') on intracortical and corticospinal excitability.

Methods: Eleven healthy subjects underwent three 10 minutes stimulation sessions: $10 \mathrm{~Hz}$ rPMS alone, applied in trains of 5 stimuli every 10 second (60 trains) on the extensor carpi radialis (ECR) muscle; rTMS alone at an intensity $120 \%$ of ECR threshold, applied at a frequency of $0.1 \mathrm{~Hz}(60$ stimuli) and magnetic PAS, i.e., paired rPMS and rTMS. We recorded motor evoked potentials (MEPs) from ECR and first dorsal interosseous (FDI) muscles. We measured resting motor threshold, motor evoked potentials (MEP) amplitude at $120 \%$ of RMT, short intracortical inhibition (SICI) at interstimulus interval (ISI) of $2 \mathrm{~ms}$ and intracortical facilitation (ICF) at an ISI of $15 \mathrm{~ms}$ before and immediately after each intervention.

Results: Magnetic-PAS, but not rTMS or rPMS applied separately, increased MEP amplitude and reduced SICI in ECR but not in FDI muscle.

Conclusion: Magnetic-PAS can increase corticospinal excitability and reduce cortical inhibition in the specific area of cortical representation of the stimulated muscle.

Significance: Application of magnetic-PAS might be relevant for motor rehabilitation.

Key words: magnetic paired associative stimulation, repetitive peripheral magnetic stimulation, motor cortex plasticity, motor cortex excitability. 


\section{INTRODUCCION}

Peripheral electrical stimulation (PES) over muscles or nerves is commonly used as an intervention to facilitate plastic changes in the motor cortex and spinal cord, enhancing recovery of motor functions (Chipchase et al 2011). Stefan et al (2000) first reported that pairedassociative stimulation (PAS), which consists of repetitive low-frequency PES of the median nerve followed by TMS of the contralateral M1 cortex, induced a transient increase in MEP amplitude at rest and in the duration of the cortical silent period (CSP) during contraction. These changes were consistently dependent on the timing of magnetic stimulation after the afferent input (Stefan et al 2000). Because PAS did not alter the excitability of spinal motor neurons or the MEP evoked by brainstem stimulation, associative long-term potentiation (LTP)-like phenomena in the M1 cortex were proposed as the underlying mechanism of PAS (Stefan et al 2000). The majority of PAS studies applied PES at suprathreshold intensities, inducing a muscle response (Kennedy and Carson 2008, Tecchio et al 2008, Litvak et al 2007, Sale et al 2007, Wolters et al 2003, Ridding and Uy 2003, Stefan et al 2000). This creates a secondary sensory influx, arising from muscle contraction, which could contribute to modulate M1 excitability (Shitara et al 2013). In fact, enhanced M1 excitability has been observed when peripheral stimuli preferentially activated proprioceptive afferents, carrying information from mechanoreceptors, muscle spindles and deep connective tissue sensors, but not when cutaneous afferents were preferentially stimulated (Kujirai et al 2006, Litvak et al 2007). Therefore, using peripheral magnetic (PMS) instead of electrical stimulation for activation of the afferent fibers would seem not to imply any difference. However, high frequency repetitive peripheral magnetic stimulation (rPMS) may itself alter corticospinal and intracortical excitability (Krause et al 2005, Krause and Straube 2008). Here, we hypothesized that the nonelectrical peripheral muscle stimulation such as rPMS which is non-painful stimulation followed by TMS could alter corticospinal excitability. The current study will investigate changes in corticospinal and intracortical excitability related to the combined application of rPMS and magnetic stimulation of the motor cortex (magnetic-PAS), in comparison to peripheral and central magnetic stimulation protocols applied alone.

\section{METHODS}

The study protocol was approved by the Research Ethics Committee of the Institute Guttmann and was conducted in accordance with the Declaration of Helsinki (World Medical 2013).

\section{Participants}

Eleven healthy subjects (5men, 6 women; mean age=36.18 \pm 10.27 years, age range: $26-64$ years; right-handed, according to the Edinburgh handedness inventory) were included in the study after 
signing written informed consent for participation in research.

\section{Experimental design and protocol}

All participants underwent 3 non-invasive stimulation protocols (rPMS, rTMS and magnetic$\mathrm{PAS}_{25}$ ), fully described below. Protocols were applied in separate visits, each spaced one-week apart. Corticospinal and intracortical excitability were assessed before and immediately after each stimulation session.

\section{Motor cortex excitability evaluation}

Changes in corticospinal and intracortical excitability were evaluated using TMS protocols. Peripheral magnetic stimulation was performed with the MagPro® (Medtronic Inc., Minneapolis, MN, USA) and delivered through a figure-of-eight coil (outer diameter of each wing $9.5 \mathrm{~cm}$ ). Subjects were seated in a comfortable chair, resting their forearms in a pronation posture on a desk in front of them and were asked to stay relaxed but awake throughout experiments. Silver/silver chloride $(\mathrm{Ag} / \mathrm{AgCl})$ disc electrodes (outer diameter of $0.9 \mathrm{~cm}$ ) were attached over the belly of the extensor carpi radialis (ECR) and first dorsal interosseous (FDI) muscles of the dominant limb, with the cathode proximal and the anode approximately $2 \mathrm{~cm}$ distally. A figure-of-eight coil was held tangentially to the scalp over the motor area of the dominant hand in the optimal position for activating the ECR, with the handle pointing backward and laterally at an angle of $45^{\circ}$ to the sagittal plane to generate a posterior-to-anterior current flow in the brain. MEPs were recorded with a conventional EMG machine (Medelec Synergy, Oxford Instruments; Surrey, England). The EMG signal was amplified using a band-pass of $3 \mathrm{~Hz}-10 \mathrm{kHz}$, amplitude sensitivity of $0.1-0.5 \mathrm{mV}$ and epochs of $100 \mathrm{~ms}$ sweep duration. They were stored in a Synergy computer for offline analysis.

The following parameters were measured before and after the neuromodulation intervention: 1. Resting motor threshold (RMT), which was defined as the lowest intensity of TMS that evoked an MEP greater than $50 \mu \mathrm{V}$ peak-to-peak amplitude in the resting target muscle in at least 5 of 10 consecutive trials, 2. Mean amplitude of MEPs using single pulse TMS at 120\% of RMT. During recordings, we marked the hot point over scalp for the biggest MEP of ECR and we also marked the localization of superficial electrodes on the belly of ECR. We used those points for stimulation and recording of MEP in ECR, 3. Short intracortical inhibition (SICI) and intracortical facilitation (ICF) using paired-pulse TMS with a subthreshold conditioning stimulus (80\% of RMT) and a suprathreshold test stimulus (120\% of RMT) at interstimulus interval (ISI) of $2 \mathrm{~ms}$ for SICI and 15ms for ICF (Kujirai et al 1993). For consistency 15 trials per test have been recorded.

The order of recordings was kept consistent for all subjects and experimental conditions as follows: RMT, MEP, SICI, and ICF. 


\section{Experiment conditions}

1) Repetitive peripheral magnetic stimulation (rPMS) was applied over the dominant ECR with the forearm placed in a neutral posture at rest on the desk, using the Magstim stimulator (Magstim, Whitland, Dyfed, UK) and a figure-of-eight-shaped coil (outer diameter of each wing: $9.5 \mathrm{~cm}$ ) centered over the muscle belly. The protocol consisted of application of trains of 5 stimuli delivered at intensity $70 \%$ (to produce dorsiflexion of the wrist). Bursts were at $10 \mathrm{~Hz}$ every 10 seconds for 10 minutes (60 trains-total 300stimuli).

2) Low frequency transcranial magnetic stimulation (rTMS) of the M1 cortex with the coil in the postero-anterior direction over the scalp site from which we recorded biggest MEP in the ECR muscle. Stimuli were of low frequency $(0.1 \mathrm{~Hz})$ suprathreshold intensity $(120 \%$ of RMT), for a total of 10 minutes, using a MagPro® (Medtronic Inc., Minneapolis, MN, USA).

3) Magnetic-PAS 25 employed paired rPMS and rTMS, as described above, in such a way that rTMS was delivered $25 \mathrm{~ms}$ after the end of each peripheral magnetic train to coincide with the arrival of the afferent volley at the motor cortex (Ridding and Taylor 2001). A specific script that allowed combining rPMS and rTMS was designed using Spike2 software (Cambridge Electronic Design Limited, Cambridge)

All volunteers were studied in the morning, between 09:00 and 12:00. Subjects were requested to stay awake and, in order to maintain their attention, they were asked to count the number of stimuli they received.

\section{Data and Statistical analysis}

We measured the peak-to-peak amplitude of MEP $(\mu \mathrm{V})$ of the ECR and FDI muscles in each recording then we calculated the mean amplitude of MEPs for each individual before and after each intervention. The SICI and ICF were calculated as percentage changes in the mean amplitude of MEP evoked by paired-pulse TMS from MEP evoked by single-pulse TMS at rest.

The two-way repeated measures ANOVAs with the factors "time" (pre- and post-intervention) and "protocol" (magnetic-PAS, rPMS and rTMS) was used to assess the effects of different interventions on RMT and MEP, and a Bonferroni adjustment was applied for multiple comparisons. The Greenhouse-Geisser correction was used when the sphericity assumption was violated. A repeated measures multivariate ANCOVA was employed to evaluate the effects of neurostimulation protocols on SICI and ICF controlling for individual differences of baselines MEP. A Student's paired t test was conducted for post hoc analysis. -

Data are expressed as mean and standard deviation and the level of significance was set at $\mathrm{p}<0.05$ for all tests. 


\section{RESULTS}

All subjects tolerated well the study. None refereed pain or unpleasant sensation related to central or peripheral stimuli.

\section{Modulation of Corticospinal Excitability}

There were no significant differences between $\operatorname{RMT}(F(2.0,20.0)=0.91, p=0.42)$ or $\mathrm{MEP}$ amplitude $(F(2.0,20.0)=0.01, p=0.99)$ at baseline (Table 1$)$. The stimulation protocols increased the corticospinal excitability as shown by significant protocol $\mathrm{x}$ time interaction $(F(2.0,20.0)=$ 6.82, $p=0.006$ ). Specifically, ECR MEP amplitude significantly increased after magnetic-PAS ( $\mathrm{p}=0.006)$, but not after rPMS $(\mathrm{p}=0.84)$ or $\mathrm{rTMS}(\mathrm{p}=0.40)$ applied alone. The neurostimulation protocols did not induce changes in RMT as indicated by non-significant effect of protocol $(F(2.0$, $20.0)=0.30, p=0.42)$ and time $(F(1.0,10.0)=1.40, p=0.26)$ or protocol $\mathrm{x}$ time interaction $(F(2.0$, 20.0) $=0.91, p=0.42$ ) (Table 1, figure 1).

\section{Modulation of Short Intracortical Inhibition and Facilitation}

Baseline measure of intracortical excitability were not different between experimental conditions $[F(2.0,20.0)=0.30, p=0.07$ for SICI and $F(2.0,20.0)=0.79, p=0.47)$ for ICF $]$. The application of magnetic-PAS led to a decrease in cortical inhibition as revealed by significant protocol $\mathrm{x}$ time interaction $(F(2.0,14.0)=5.30, p=0.02)$ with reduction of SICI $(\mathrm{p}=0.03)$ after magnetic-PAS but not after rPMS $(\mathrm{p}=0.56)$ or rTMS $(\mathrm{p}=0.18)$. Furthermore, there were no statistically significant changes of ICF $(F(2.0,14.0)=0.73, p=0.50)$ when comparing between different experimental conditions (Table 1, figure 2).

In the FDI, no significant changes of $\operatorname{RMT}(F(2.0,20.0)=0.91, p=0.42)$, MEP amplitude $(F(2.0$, $20.0)=0.80, p=0.46), \operatorname{SICI}(F(2.0,14.0)=0.72, p=0.50)$ or $\operatorname{SCIF}(F(2.0,14.0)=0.56, p=0.59)$ were observed (Table 1).

\section{DISCUSSION}

To our knowledge, this is the first study to examine changes in corticospinal and intracortical excitability following magnetic-PAS. We have demonstrated that these changes were specifically related to synchronous stimulation of M1 and the muscle receiving rPMS. The results indicate that the enhancement of M1 cortex excitability induced by magnetic-PAS may be somatotopically specific, as shown by augmented corticospinal excitability and reduced intracortical inhibition limited to the stimulated muscle. The effects were not present when the peripheral and central nervous system stimulation protocols were applied separately. 
Our findings are consistent with previous reports of increased M1 cortex excitability when either conventional (Sale et al 2007, Stefan et al 2000, Wolters et al 2003) or magnetic-PAS was employed (Ridding and Taylor 2001). MEP amplitude results from corticospinal and spinal-segmental contributions to spinal cord motorneurons. Evidence from individuals who had an electrode implanted in the cervical epidural space indicate that $\mathrm{PAS}_{25}$ might induce LTP-like synaptic plasticity of the M1 cortex output neurons as the associative stimulation enhanced late but not early corticospinal volleys (Di Lazzaro et al 2011). Although local modulation of spinal and supraspinal circuitry induced by TMS and PES may play a role in corticospinal facilitation (Meunier et al 2007, Taylor and Martin 2009), this form of heterotopic neuroplasticity is considered to occur in the M1 cortex because PES and rTMS synchronized at the spinal cord failed to modulate corticospinal function (McGie et al 2014). However, other factors such as baseline cortical inhibitory states (Murase et al 2015), surround motor cortex inhibition (Belvisi et al 2014) and the quality of the sensory afferents (Kennedy and Carson 2008, Litvak et al 2007, Sale et al 2007, Stefan et al 2000, Tecchio et al 2008) could also interfere in PAS-related facilitatory mechanism.

In this context, the rPMS could present some advantages over PES. Compared to the PES, which provides a strong cutaneous inflow (including afferents to lemniscal and spino-thalamic pathways), rPMS generates a significant proprioceptive inflow (directly via recruitment of 1a-afferents or indirectly via muscle contraction and joint angular displacement). Another advantage is that rPMS is painless and well tolerated (Beaulieu and Schneider 2015). To our knowledge there is only one study that showed successful M1 cortex facilitation in healthy subjects after 30 minutes of paired central suprathreshold magnetic stimuli and peripheral nerve or muscle (FDI) stimulation (Ridding and Taylor 2001), but no control conditions were included in the study.

\section{Intracortical inhibition}

The effects of PAS on intracortical inhibition and facilitation have been investigated previously. In the current study, we found that the combination of peripheral and central magnetic stimulation induced an enhancement of M1 cortex excitability associated with reduction of SICI with no changes in ICF. SICI reflects GABA-A mediated intracortical inhibition (Ziemann et al., 1996). Modulation of intracortical inhibitory mechanisms play a determining role in PAS-related plasticity as suggested by suppression of MEP modulation when a conditioning subthreshold magnetic stimulus was delivered $2 \mathrm{~ms}$ (with inhibitory effect on cortical excitability) before the test stimuli (Elahi et al 2012). In addition, a strong baseline SICI predicted good neuromodulatory response to PAS (Murase et al 2015, Russmann et al 2009). With the acute deafferentation or ischaemic block, intracortical inhibition is reduced if low frequency TMS is used to enhance the cortical changes (Ziemann et al. 1998a). It is thought that the reduction in inhibition may have a role to play in MEP 
facilitation (Ziemann et al. 1998). However, low-frequency TMS alone did not cause changes in SICI, but magnetic-PAS did. We think that changes in SICI could be due to the combination of specific effects from rPMS-generated inputs on cortical excitability, possibly mediated by weaker GABA-ergic inhibition (Carson and Kennedy 2013).

\section{Intracortical facilitation}

Relatively little is known about the mechanism underlying ICF. ICF reflects mainly Glutamatergic N-methyl- D-asparate (NMDA) -mediated intracortical facilitation (Schwenkreis et al 1999, Ziemann et al 1996-1998) that engages neuronal circuits independent from those mediating SICI. In our study, magnetic-PAS did not cause changes in intracortical facilitation, which means that magnetic-PAS did not induce changes in NMDA level and which is in agreement with previous reports (Murase et al 2015, Roy et al 2007).

\section{Role of peripheral magnetic stimulation}

The neuromodulatory effects of the peripheral electric stimulation depend on the intensity and frequency of stimulation. High-frequency subthreshold (Tinazzi et al 2005) and low frequency suprathreshold but not low frequency subthreshold electrical stimulation (Golaszewski et al 2012) induced facilitatory effects on the motor cortex with no effects on spinal excitability (Golaszewski et al 2012, Tinazzi et al 2005). Commonly, the intensity of peripheral stimulation employed in PAS protocols (Kennedy and Carson 2008, Litvak et al 2007, Sale et al 2007, Stefan et al 2000, Tecchio et al 2008, Wolters et al 2003) was defined in relation to the elicitation of visible muscle twitches, which requires delivery of suprathreshold electrical stimuli. Therefore, in addition to activation of direct cutaneous ascending afferents by electrical stimulation, a secondary volley arising from muscle can have neuromodulatory effect on the spinal and supraspinal circuits (Shitara et al 2013). Compared to electrical nerve stimulation, rPMS generates a greater proprioceptive inflow through recruitment of muscle and joint afferents (Beaulieu and Schneider 2015). High frequency rPMS is known to induce robust neuroplastic changes at cortical level. Suprathreshold, $20 \mathrm{~Hz}$ rPMS (2000 pulses in a single session) applied over cervical roots $(\mathrm{C} 7 / \mathrm{C} 8)$ led to prolongation of the CSP (Krause et al 2005, Krause and Straube 2008), increase amplitude of MEP and raised SICI (Krause and Straube 2008). However, the facilitation of corticospinal and intracortical excitability induced by rPMS is sensitive to the frequency and the total number of applied magnetic impulses with higher frequency and greater number of stimuli inducing more robust effects (Gallasch et al 2015).

\section{Limitations and future directions}

The rPMS protocol used in the current study included only 300 magnetic pulses at $10 \mathrm{~Hz}$ and applied over the muscle, which had no effect on corticospinal or intracortical excitability. Applying 
rPMS over the nerve root could be an alternative to induce more effective changes in MEP and SICI. Our research was conducted in healthy individuals and therefore cannot be extrapolated to patients with damage in the central nervous system. However, intracortical inhibitory activity is known to contribute to persistent abnormal motor behavior in the paretic limb (Hummel et al 2009) being a determining factor of the reorganization pattern and motor cortex plasticity after stroke (Hummel et al 2009, Liepert et al 2006). Magnetic-PAS could present some advantages over conventional PAS protocols for motor rehabilitation being non-invasive, non-painful peripheral stimulation and as suggested by our finding of decreased intracortical inhibition. Because the baseline SICI seems to be a determining factor of the modulatory effects of PAS (Murase et al 2015, Russmann et al 2009) future studies should evaluate the effects of GABA-ergic medication on motor cortex plasticity related with magnetic-PAS. The neuromodulatory effects of rPMS are sensitive to the frequency and the total number of applied stimuli (Gallasch et al 2015, Krause et al 2005, Krause and Straube 2008) therefore future research studies should also investigate whether employing higher frequencies and larger durations of rPMS in magnetic-PAS protocols could enhance M1 cortex facilitation.

\section{SIGNIFICANCE}

Short sessions of magnetic-PAS can modulate the excitability of the M1 cortex, leading to increased corticospinal excitability and decreased cortical inhibition in the stimulated muscle. This new paradigm may offer a useful therapeutic option for motor rehabilitation.

\section{Conflict of Interest Statement: None.}

\section{Acknowledgement}

This research was supported in part by grants from Foundation La Marató TV3 PI110932 to HK.

\section{REFERENCES}

Beaulieu LD, Schneider C. Repetitive peripheral magnetic stimulation to reduce pain or improve sensorimotor impairments: A literature review on parameters of application and afferents recruitment. Neurophysiol Clin 2015; 45: 223-37

Belvisi D, Kassavetis P, Bologna M, Edwards MJ, Berardelli A, Rothwell JC. Associative plasticity in surround inhibition circuits in human motor cortex. Eur J Neurosci 2014; 40: 3704-10 
Carson RG, Kennedy NC. Modulation of human corticospinal excitability by paired associative stimulation. Front Hum Neurosci 2013; 7: 823

Chipchase LS, Schabrun SM, Hodges PW. Peripheral electrical stimulation to induce cortical plasticity: a systematic review of stimulus parameters. Clin Neurophysiol 2011; 122: 456-63

Di Lazzaro V, Dileone M, Pilato F, Capone F, Musumeci G, Ranieri F, et al. Modulation of motor cortex neuronal networks by rTMS: comparison of local and remote effects of six different protocols of stimulation. J Neurophysiol 2011; 105: 2150-6

Elahi B, Gunraj C, Chen R. Short-interval intracortical inhibition blocks long-term potentiation induced by paired associative stimulation. J Neurophysiol 2012; 107: 1935-41

Gallasch E, Christova M, Kunz A, Rafolt D, Golaszewski S. Modulation of sensorimotor cortex by repetitive peripheral magnetic stimulation. Front Hum Neurosci 2015; 9: 407

Golaszewski SM, Bergmann J, Christova M, Kunz AB, Kronbichler M, Rafolt D, et al. Modulation of motor cortex excitability by different levels of whole-hand afferent electrical stimulation. Clin Neurophysiol 2012;123: 193-9

Hummel FC, Steven B, Hoppe J, Heise K, Thomalla G, Cohen LG, et al. Deficient intracortical inhibition (SICI) during movement preparation after chronic stroke. Neurology 2009; 72: $1766-72$

Kennedy NC, Carson RG. The effect of simultaneous contractions of ipsilateral muscles on changes in corticospinal excitability induced by paired associative stimulation (PAS). Neurosci Lett 2008; 445: 7-11

Krause P, Foerderreuther S, Straube A. Effects of conditioning peripheral repetitive magnetic stimulation in patients with complex regional pain syndrome. Neurol Res 2005; 27: 412-7

Krause P, Straube A. Peripheral repetitive magnetic stimulation induces intracortical inhibition in healthy subjects. Neurol Res 2008; 30: 690-4

Kujirai K, Kujirai T, Sinkjaer T, Rothwell JC. Associative plasticity in human motor cortex during voluntary muscle contraction. J Neurophysiol 2006; 96: 1337-46

Kujirai T, Caramia MD, Rothwell JC, Day BL, Thompson PD, Ferbert A, et al. Corticocortical inhibition in human motor cortex. J Physiol 1993; 471: 501-19

Liepert J, Haevernick K, Weiller C, Barzel A. The surround inhibition determines therapy-induced cortical reorganization. Neuroimage 2006; 32: 1216-20

Litvak V, Zeller D, Oostenveld R, Maris E, Cohen A, Schramm A, Gentner R, et al. LTP-like changes induced by paired associative stimulation of the primary somatosensory cortex in humans: source analysis and associated changes in behaviour. Eur J Neurosci 2007; 25: 2862-74.

McGie SC, Masani K, Popovic MR. Failure of spinal paired associative stimulation to induce 
neuroplasticity in the human corticospinal tract. J Spinal Cord Med 2014; 37: 565-74

Meunier S, Russmann H, Simonetta-Moreau M, Hallett M. Changes in spinal excitability after PAS. J Neurophysiol 2007; 97: 3131-5

Murase N, Cengiz B, Rothwell JC. Inter-individual variation in the after-effect of paired associative stimulation can be predicted from short-interval intracortical inhibition with the threshold tracking method. Brain Stimul 2015; 8: 105-13.

Quartarone A, Rizzo V, Bagnato S, Morgante F, Sant'Angelo A, Girlanda P, et al. Rapid-rate paired associative stimulation of the median nerve and motor cortex can produce long-lasting changes in motor cortical excitability in humans. J Physiol 2006; 575: 657-70.

Ridding MC, Uy J. Changes in motor cortical excitability induced by paired associative stimulation. Clin Neurophysiol. 2003 ;114:1437-44.

Ridding MC, Taylor JL. Mechanisms of motor-evoked potential facilitation following prolonged dual peripheral and central stimulation in humans. J Physiol 2001; 537: 623-31

Roy FD, Norton JA, Gorassini MA. Role of sustained excitability of the leg motor cortex after transcranial magnetic stimulation in associative plasticity. J Neurophysiol 2007; 98: 657-67

Russmann H, Lamy JC, Shamim EA, Meunier S, Hallett M. Associative plasticity in intracortical inhibitory circuits in human motor cortex. Clin Neurophysiol 2009; 120: 1204-12

Sale MV, Ridding MC, Nordstrom MA. Factors influencing the magnitude and reproducibility of corticomotor excitability changes induced by paired associative stimulation. Exp Brain Res 2007; 181: 615-26

Schwenkreis P, Witscher K, Janssen F, Addo A, Dertwinkel R, Zenz M, et al. Influence of the Nmethyl-D-aspartate antagonist memantine on human motor cortex excitability. Neurosci Lett 1999; 270: 137-40

Shitara H, Shinozaki T, Takagishi K, Honda M, Hanakawa T. Movement and afferent representations in human motor areas: a simultaneous neuroimaging and transcranial magnetic/peripheral nerve-stimulation study. Front Hum Neurosci 2013; 7: 554

Stefan K, Kunesch E, Cohen LG, Benecke R, Classen J. Induction of plasticity in the human motor cortex by paired associative stimulation. Brain 2000;123 Pt 3: 572-84

Taylor JL, Martin PG. Voluntary motor output is altered by spike-timing-dependent changes in the human corticospinal pathway. J Neurosci 2009; 29: 11708-16

Tecchio F, Zappasodi F, Pasqualetti P, De Gennaro L, Pellicciari MC, Ercolani M, et al. Age dependence of primary motor cortex plasticity induced by paired associative stimulation. Clin Neurophysiol 2008; 119: 675-82

Tinazzi M, Zarattini S, Valeriani M, Romito S, Farina S, Moretto G, et al. Long-lasting modulation of human motor cortex following prolonged transcutaneous electrical nerve stimulation (TENS) of forearm muscles: evidence of reciprocal inhibition and facilitation. Exp Brain 
Res 2005; 161: 457-64

Wolters A, Sandbrink F, Schlottmann A, Kunesch E, Stefan K, Cohen LG, et al. A temporally asymmetric Hebbian rule governing plasticity in the human motor cortex. J Neurophysiol 2003; 89: 2339-45

World Medical A. World Medical Association Declaration of Helsinki: ethical principles for medical research involving human subjects. JAMA 2013; 310: 2191-4

Ziemann U, Rothwell JC, Ridding MC. Interaction between intracortical inhibition and facilitation in human motor cortex. J Physiol 1996; 496 ( Pt 3): 873-81.

Ziemann U, Corwell B, Cohen LG. Modulation of plasticity in human motor cortex after forearm ischemic nerve block. Journal of Neuroscience 1998; 18, 1115-1123.

Figure legends:

Figure 1. Modulation of MEP at ECR muscle. The thin lines represent individual MEP amplitude, and the thick line represent the average MEP amplitude in each experimental group.

Figure 2. Modulation of SICI at ECR muscle. The thin line represents individual SICI, and the thick line represents the average SICI in each experimental group. 